279-89.

${ }^{125}$ Fouli MA, Sayed AA, Badawi AA. Release of drugs from microcapsules of methacrylate polymers. Intern J Pharm I983; I4:95-I02.

${ }^{126}$ Lee PI. Dimensional changes during drug release from a glassy hydrogel matrix. Polym Commun $1983 ; 24: 45-$ 7.

127 Lee PI. Effect of non-uniform initial drug concentration distribution on the kinetics of drug release from glassy hydrogel matrices. Polymer I 984;25:973-8.

${ }^{128}$ Bahadir M. Pestizide Polymere Untersuchung pestizider 'controlled release' Formulierungen mit LDPE und EVA. Chemosphere 1983;12:1327-36.

${ }^{129}$ Langer RS, Hsieh DST, Brown L. Polýmeric delivery systems for macromolecules. Washington DC: American Chemical Society, I982:95-I05. (ACS Symposium Series. Vol. I86.)

${ }^{130}$ Baker RW, Lonsdale HK. Controlled release: mechanisms and rates. In: Tanquary AC, Lacey RE, eds. Advances in Experimental Medicine and Biology. Vol. 47. New York: Plenum Press, 1974:15-7I .

131 Higuchi T. Rate of release of medicaments from ointment bases containing drugs in suspension. J Pharm Sci $1961 ; 50: 874-5$.

${ }^{132}$ Chien YW, Lau EPK. Controlled drug release from polymeric delivery devices. IV: In vitro-in vivo correlation of subcutaneous release of Norgestomet from hydrophilic implants. J Pharm Sci 1976;65:488-92.

${ }^{133}$ De Leede LGJ. Rate-controlled and site-specific rectal drug delivery. Leiden: State University of Leiden, I 983 . Dissertation.

${ }^{134}$ Cardinal JR. Drug release from matrix devices. In: Anderson JH, Kim SW, eds. Recent Advances in Drug Delivery Systems. Proceedings of an International Symposium. New York: Plenum Publishing Corporation, $1984: 229-48$.

135 Cardinal JR, Kim SW, Song S, Lee ES, Kim SH. Controlled release drug delivery systems from hydrogels: Progesterone release from monolithic, reservoir, combined reservoir monolithic and monolithic devices with rate controlling barriers. AIChE Symposium. Series $198 \mathrm{I} ; 77(206): 52-6 \mathrm{I}$.

136 Roseman TJ, Higuchi WI. Release of medroxyprogesterone acetate from a silicone polymer J Pharm Sci 1970;59:353-7.

${ }^{137}$ Chien YW, Lambert HJ, Lin TK. Solution-solubility dependency of controlled release of drug from polymer matrix: mathematical analysis. J Pharm Sci I975:64: $1643-7$.

${ }^{13 \times}$ Chien YW, Lambert HJ, Grant DE. Controlled drug release from polymeric devices. I. Technique for rapid in vitro release studies. J Pharm Sci 1974;63:365-9.

${ }^{139}$ Chien YW, Lambert HJ. Controlled drug release from polymeric delivery devices. II. Differentiation between partition-controlled and matrix-controlled drug release mechanisms. J Pharm Sci 1974;63:515-9.

${ }^{1+1}$ Chien YW, Mares, S, Berg J, Huber S, Lambert HJ, King KF. Controlled drug release from polymeric devices. III. J Pharm Sci I 975;64: I776-8I.

${ }^{1+1}$ Haleblian J, Runkel R, Mueller N, Christopherson J, $\mathrm{Ng} \mathrm{K}$. Steroid release from silicone elastomer containing excess drug in suspension. J Pharm Sci 197I; 60:541-4.

${ }^{142}$ Chandrasekaran SK, Paul DR. Dissolution controlled transport from dispersed matrixes. J Pharm Sci I 982;71: 1399-402.

${ }^{143}$ Brooke D, Washkun J. Zero-order drug delivery systems, theory and preliminary investigation. J Pharm Sci $1977 ; 66: 159-62$.

it Thomas NL, Windle RH. A theory of case II diffusion. Polymer 1982;23:529-42.

14.5 Thomas N, Windle AH. Discontineous sharp change associated with case II transport of methanol in thin sheets of PMMA. Polymer 1977;18:1 195.

${ }^{146}$ Peterlin A. Diffusion in a glassy polymer with discontinuous swelling. II. Concentration distribution of diffusant as function of time. Makromol Chem 1969; 124 : 136-42.

${ }^{147}$ Hopfenberg HB, Hsu KC. Swelling-controlled, constant rate delivery systems. Polym Eng Sci I 978; 1 8: I I 86-9 I.

1+8 Hopfenberg HB. A mechanistic interpretation of swelling-controlled, constant rate delivery systems. AIChE Symposium Series $1981 ; 77(206): 37-41$.

${ }^{1.4}$ Korsmeyer RW, Gurny R, Doelker E, Buri P, Peppas NA. Mechanisms of solute release from porous hydrophilic polymers. Int J Pharm 1983;1 5:25-35.

${ }^{150}$ Good WR. Diffusion of water soluble drugs from initially dry hydrogels. Midl Macromol Monogr I $978 ; 5: 139-56$.

${ }^{151}$ O'Neil WP. Membrane systems. In: Kynodieus AF, ed. Controlled release technologies. Part 1. Boca Raton: CRC Press, I980: 157-60.

${ }^{152}$ Hurkmans JFGM, Boddé HE, Van Driel LMJ, Van Doorne $H$, Junginger HE. Skin irritation by transdermal drug delivery systems during long term application. Br J Dermatol 1985; I 12:46I-7.

Received January 1986.

Accepted for publication May 1986.

\title{
erratum
}

\section{Anthracycline antitumour agents. A review of physicochemical, analytical and stability properties}

\section{J. BOUMA, J.H. BEIJNEN, A. BULT AND W.J.M. UNDERBERG}

A mistake was made in the correction of this paper (Pharm Weekbl [Sci] 1986;8: I09-33). Due to this mistake, in the right-hand column of page 113 the lines $36-40$ were scrambled. The sentence should have read as follows: 'Additionally, the adsorption phenomenon of doxorubicin was used to clean doxorubicin liposome preparations from free doxorubicin by adsorption of the free drug onto Dowex $50 \mathrm{~W}-\mathrm{X}_{4}$ '. 